immunoregulatory $\mathrm{T}$ cells that control autoimmune diabetes. Immunity. 12:431-440.

40. Tang, Q., et al. 2006. Visualizing regulatory T cell control of autoimmune responses in nonobese diabetic mice. Nat. Immunol. 7:83-92.

41. Fillatreau, S., Sweenie, C.H., McGeachy, M.J., Gray, D., and Anderton, S.M. 2002. B cells regulate autoimmunity by provision of IL-10. Nat. Immunol. 3:944-950.

42. Goetz, M., et al. 2007. Exacerbation of ulcerative colitis after rituximab salvage therapy. Inflamm. Bowel Dis. 13:1365-1368.

43. Maki, T., Ichikawa, T., Blanco, R., and Porter, J. 1992. Long-term abrogation of autoimmune diabetes in nonobese diabetic mice by immunotherapy with anti-lymphocyte serum. Proc. Natl. Acad. Sci. U. S. A. 89:3434-3438.

44. Belghith, M., et al. 2003. TGF-beta-dependent mechanisms mediate restoration of self-tolerance induced by antibodies to CD3 in overt autoim- mune diabetes. Nat. Med. 9:1202-1208.

45. Mohty, M. 2007. Mechanisms of action of antithymocyte globulin: T-cell depletion and beyond. Leukemia. 21:1387-1394.

46. Herold, K.C., et al. 2002. Anti-CD3 monoclonal antibody in new-onset type 1 diabetes mellitus. N. Engl. J. Med. 346:1692-1698.

47. Immune Tolerance Network. Clinical trials for newly diagnosed type 1 diabetes. http://www. newonsetdiabetes.org/trials.html.

\title{
Every allograft needs a silver lining
}

\author{
Alan G. Contreras and David M. Briscoe
}

Transplantation Research Center and Division of Nephrology, Children's Hospital Boston, Boston, Massachusetts, USA. Department of Pediatrics, Harvard Medical School, Boston, Massachusetts, USA.

\begin{abstract}
The development of chronic allograft rejection is based on the hypothesis that cumulative, time-dependent tissue injury eventually leads to a fibrotic response. In this issue of the JCI, Babu and colleagues found that alloimmune-mediated microvascular loss precedes tissue damage in murine orthotopic tracheal allografts (see the related article beginning on page 3774 ). The concept that injury to the endothelium may precede airway fibrosis suggests that interventions to maintain vascular integrity may be important, especially in the case of lung transplantation. Further, for all solid organ allografts, it is possible that the key to long-term allograft survival is physiological vascular repair at early times following transplantation.
\end{abstract}

\section{Clues to mechanisms underlying long-term allograft survival}

The major obstacle to the long-term survival of lung transplant recipients is the development of the bronchiolitis obliterans syndrome (BOS), which typically occurs in up to $60 \%$ of patients who survive five years (1). BOS is considered a major posttransplant complication and is characterized by a progressive luminal airway narrowing and histological evidence of fibrosis. Pathological features of BOS suggest that injury and inflammation of epithelial cells and subepithelial structures within small airways lead to the fibrosis response. Moreover, it is thought that repetitive injury in lung allografts, as in other solid organ transplants (e.g., kidney) $(2,3)$, may elicit cumulative, time-dependent damage and result in cellular atrophy and chronic interstitial fibrosis (1). According to this paradigm, prevention or inhibition of injury (using time-dependent therapies) should result in long-term graft survival (4).

Nonstandard abbreviations used: BOS, bronchiolitis obliterans syndrome.

Conflict of interest: David M. Briscoe has received research funding and/or has served as a consultant to the Roche Organ Transplantation Research Foundation and Wyeth Pharmaceuticals.

Citation for this article: J. Clin. Invest. 117:3645-3648 (2007). doi:10.1172/JCI34238.
Nevertheless, for all solid organ allografts, an underappreciated aspect of the injury process is its association with repair. It has been proposed that augmenting physiological repair, even in the face of injury, will ensure that the end result of injury is limited $(5,6)$. On the other hand, if the repair process is inhibited, then even milder forms of injury have the potential to result in extensive tissue damage. Therefore, it is possible that an understanding of repair is key to long-term allograft survival.

In this issue of the JCI, Babu et al. set out to address whether the maintenance of microvasculature integrity is sufficient to sustain lung allograft function and to prevent the development of fibrosis/chronic rejection (7). These authors used a murine orthotopic tracheal transplantation model and found that alloimmune-mediated microvascular loss results in hypoxia, which precedes tissue damage and the development of intragraft fibrosis in the recipient trachea. If endothelial cell injury was inhibited (by limiting ongoing inflammation) and the microvasculature was maintained intact, physiological remodeling occurred and allograft tissue morphology in these animals returned to normal (Figure 1). Therefore, in general, once an allograft is revascularized and is functioning, protect- ing the vasculature and/or enabling physiological homeostatic repair of the microvasculature will prevent tissue fibrosis.

\section{Role of microvascular repair in the preservation of allografts}

In the context of transplantation, it should be emphasized that microvascular endothelial cells are very susceptible to injury, including changes in oxygen tension (hypoxic injury), reperfusion injury, and oxidative stress, as well as persistent episodes of silent rejection (8). These repetitive insults clearly target the microvasculature and create a circumstance whereby endothelial loss and/or damage will typically occur $(9,10)$. Within allografts, the loss of the microvasculature results in impaired delivery of oxygen and nutrients to key organ-specific cells, such as columnar epithelial cells in the lung or tubular epithelial cells in the kidney, which in turn results in chronic ischemia and cell death $(5,6,8,11)$. If vascular integrity is coincidently compromised, recovery may not be complete and/or may not occur, and cellular atrophy will lead to progressive functional tissue loss. The implication of these observations is that the limiting factor in the allograft injury process is inefficient microvascular repair. This concept may be particularly relevant following lung transplantation, where bronchial artery vascular reanastomosis is often not done, and thus vascular integrity may be easily compromised at early times as a result of alloimmune attack. Indeed, microvascular injury has been documented in association with rejection (12), and replacement of endothelial cells correlates with degrees of injury in human allografts $(8,13,14)$. Therefore, one must assume that the health 


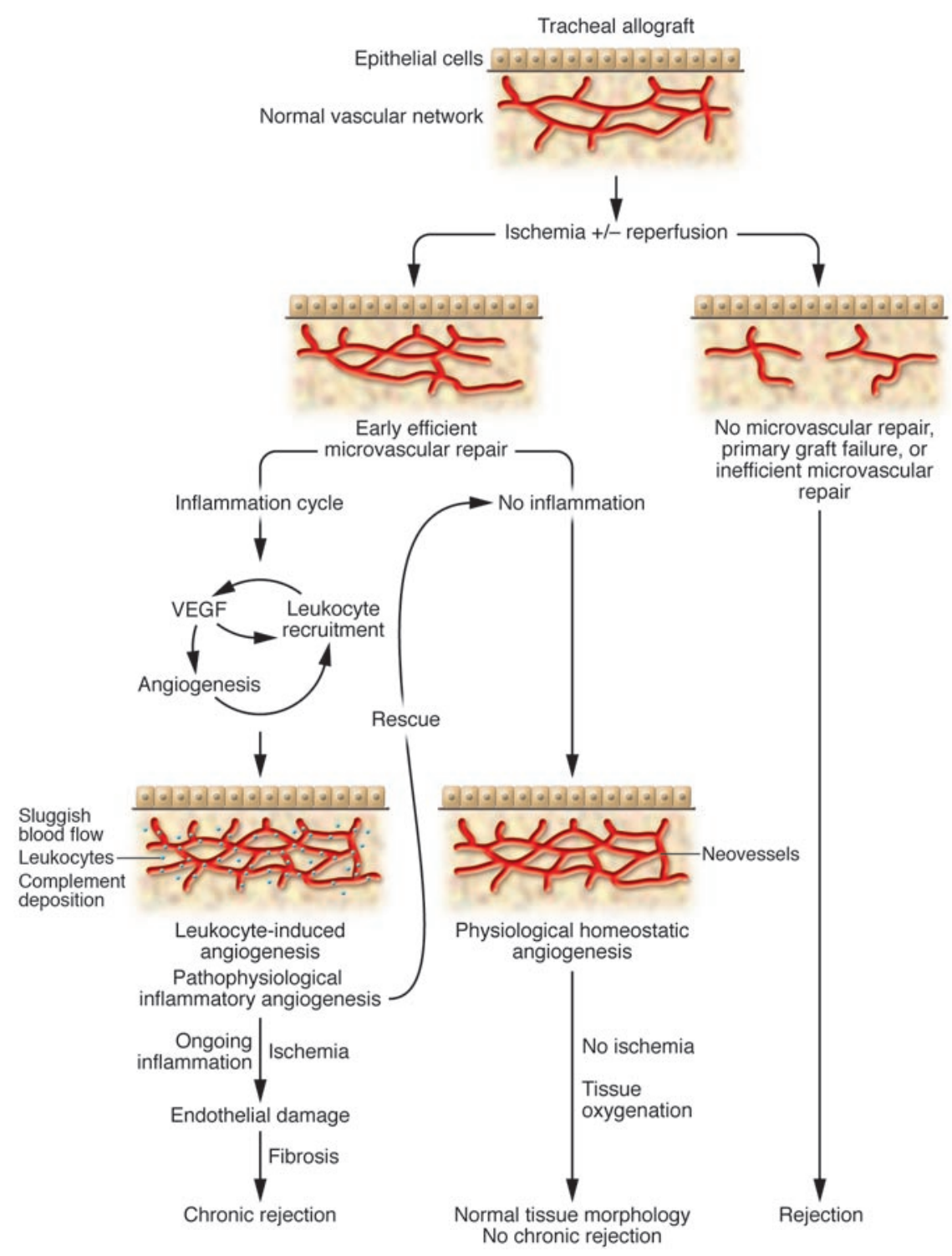

of an allograft can only be maintained if early physiological turnover of endothelial cells is efficient.

\section{The leukocyte-induced neovascular response: road to repair or a means to destruction?}

In their seminal studies evaluating the process of leukocyte-induced angiogenesis, Sidky and Auerbach injected either allogeneic or syngeneic spleen cells into the skin of nude mice $(15,16)$. They found that allogeneic, but not syngeneic, leukocytes mediated an angiogenic response. These classical studies clearly demonstrated that the alloactivated leukocyte produces factors that facilitate neovascularization. Moreover, Auerbach found that allogeneic leukocyte-induced angiogenesis occurred within 3-6 days following injection and was dose-dependent inasmuch as it was enhanced with an increase in the number of injected cells (15). Therefore, it should be no surprise that angiogenesis is a component of allograft rejection. Interestingly, in this issue of the JCI, Babu et al. (7) observed a neovascularization reaction in their analysis of tracheal allografts undergoing rejection. Although they did not characterize this response from the time of transplantation (day 0), their studies clearly illustrate a prominent neovascularization reaction between days 6 and 8 after transplantation; but with ongoing inflammation, this neovascularization reaction did not persist, and it was not sufficient to sustain tissue oxygenation. Therefore, in the tracheal allograft model,

\section{Figure 1}

Microvascular injury and repair following tracheal transplantation. Following orthotopic transplantation of trachea, revascularization occurs within four days and is associated with perfusion and return of blood flow. This revascularization response involves efficient physiological anastomoses between donor and recipient microvessels. This early efficient repair and/or homeostatic angiogenesis is required for normal graft function. After reperfusion, in the absence of inflammation (such as occurs in syngrafts or immunosuppressed recipients of allografts), physiological homeostatic vascular remodeling occurs, microvascular integrity is maintained, and tissue morphology remains normal. In contrast, as leukocytes infiltrate allografts in association with rejection, pathophysiological inflammatory angiogenesis occurs and is only sufficient to sustain graft function minimally. This inflammatory angiogenesis reaction likely facilitates ongoing leukocyte recruitment and endothelial damage, eventually leading to ischemia and a cycle of microvascular injury and hypoxia that cannot sustain tissue function. The end result of this cycle is tissue fibrosis and chronic rejection. In this issue of the $\mathrm{JCl}$, Babu et al. (7) demonstrated that early physiological homeostatic repair and the absence of inflammation will sustain long-term tissue function and morphology. local tissue hypoxia and the production of hypoxia-inducible growth factors are likely the stimuli for the initial revascularization response (until day 4); but, one might conclude that it is unlikely that hypoxia mediates the later angiogenenic response. Rather, the second phase of angiogenesis is likely mediated by infiltrating allogeneic leukocytes and inflammation, analogous to Auerbach's original observations. The local tissue hypoxia may be a consequence of sluggish blood flow associated with the inflammatory reaction.

So why does this later neoangiogenic response fail to sustain allograft function? This is an important question, as this response likely occurs in association with chronic graft rejection. One possible answer to this question relates to the proin- 
flammatory nature of the leukocyte-inducible angiogenic reaction (Figure 1). Neovessels are in themselves "sticky" and facilitate the recruitment of leukocytes, in part via their expression of adhesion molecules and chemokines. Therefore, in allografts, once inflammatory angiogenesis is established, it may serve to facilitate alloimmune leukocyte recruitment at a time when clonal expansion of destructive lymphocytes is maximized. This will result in further injury. This concept is characteristic of chronic inflammatory disease processes, which have been defined as repetitive bouts of acute inflammation and tissue destruction that proceed simultaneously with attempts at repair (17). The study by Babu et al. (7) has provided some clues as to the functional role of this type of angiogenesis. The authors found that removal of allografts with intact microvessels from the recipients on day 6 and retransplantation into immunosuppressed allogeneic recipients resulted in rescue, with normal airway morphology at day 28. If, on the other hand, microvessels within the removed and retransplanted allografts were not intact, then rescue was not achieved. Therefore, one might conclude that so long as early microvascular repair occurs efficiently, then protection of homeostatic angiogenesis/microvascular repair and the interruption of leukocyte recruitment and leukocyte-induced angiogenesis within grafts at critical times after transplant will sustain allograft function.

\section{A balancing act: therapeutic considerations}

In vascularized solid organ allografts, such as kidney or heart, the early ischemia/reperfusion response can result in profound injury to the microvasculature $(6,8)$. Clearly, in part, early repair can be augmented by limiting the degree of injury (hypoxia, oxidative stress, ischemia/reperfusion, etc.), but it could also be accomplished via specific therapies, for instance, erythropoietin treatment (18). Erythropoietin can induce protective genes within vascular endothelial cells (phosphorylated Akt, Stat5, and downstream substrates such as members of the FoxO family, eNOS, and $\mathrm{Bcl} 2$, etc.) (19) and has the potential to facilitate microvascular repair following ischemia/reperfusion (20).

Growth factors such as VEGF have multiple biological properties that can facilitate both protection of the microvasculature and angiogenesis $(5,21)$. However, VEGF also facilitates leukocyte recruitment and in this manner may promote tissue injury (22). VEGF may be a prototype growth factor that facilitates the overlap between angiogenesis and inflammation (5). Consistent with studies in vascularized allografts (22), Babu et al. (7) found that blockade of VEGF did not inhibit initial revascularization, but it markedly inhibited the later leukocyteinduced response (after day 4-6). We suspect that VEGF antagonism may have had some effect on the early response (albeit subtle) to disrupt the degree of protection or cell survival pathways within endothelial cells, such that any inflammation response at later times is more destructive. Clearly there are times when VEGF may be protective and there are also circumstances, such as in inflammation, when VEGF-induced angiogenesis is not protective and may become a means to destruction. As discussed by the authors (7), perhaps VEGF expression within the tracheal allograft serves to promote leukocyte recruitment, but this inflammatory angiogenesis may only be temporarily protective. Moreover, we suggest that leukocyte-induced angiogenesis cannot promote effective repair and thus, we like to consider it "pathophysiological angiogenesis." This is the type of angiogenesis that occurs in association with chronic rejection. If VEGF/VEGF function is inhibited or if inflammation is inhibited, the cycle of leukocyte recruitment and angiogenesis will be reduced, and thus, tissue destruction will be attenuated (Figure 1). Indeed, we and others have found that graft morphology is remarkably improved and infiltrates are reduced when recipients with early chronic rejection are treated with angiogenesis inhibitors or VEGF antagonists $(5,23-25)$.

In conclusion, the study by Babu et al. (7) reported in this issue of the JCI has emphasized the importance of microvascular integrity for long-term allograft function. These data suggest that therapy that protects vascular endothelial cells from injury and/or optimizes physiological noninflammatory angiogenesis within allografts may serve to facilitate long-term allograft function. In contrast, the later pathophysiological inflammatory angiogenesis may facilitate ongoing leukocyte recruitment and injury and does not serve a protective function. Clearly, this is an area that deserves further study, as chronic rejection is the most common cause of solid organ allograft failure and to date no therapy has been successful in interrupting this process in humans. Therefore, a robust vasculature appears to be the "silver lining" that is necessary to sustain long-term allograft function.

\section{Acknowledgments}

The authors' research is supported by NIH grants R01 HL074436 and R01 AI046756, the Roche Organ Transplantation Research Foundation, an investigator-originated proposal from Wyeth Pharmaceuticals (to D.M. Briscoe), and a grant from the American Society of Transplant Surgeons (to A.G. Contreras).

Address correspondence to: David M. Briscoe, Division of Nephrology, Children's Hospital Boston, 300 Longwood Avenue, Boston, Massachusetts 02115, USA. Phone: (617) 355-6129; Fax: (617) 730-0130; E-mail: david.briscoe@childrens.harvard.edu.

1. Estenne, M., and Hertz, M.I. 2002. Bronchiolitis obliterans after human lung transplantation. Am. J. Respir. Crit. Care Med. 166:440-444.

2. Nankivell, B.J., et al. 2003. The natural history of chronic allograft nephropathy. N. Engl. J. Med. 349:2326-2333.

3. Nankivell, B.J., and Chapman, J.R. 2006. Chronic allograft nephropathy: current concepts and future directions. Transplantation. 81:643-654.

4. Chapman, J.R., O'Connell, P.J., and Nankivell, B.J. 2005. Chronic renal allograft dysfunction. J. Am. Soc. Nephrol. 16:3015-3026.

5. Reinders, M.E., Rabelink, T.J., and Briscoe, D.M. 2006. Angiogenesis and endothelial cell repair in renal disease and allograft rejection. J. Am. Soc. Nephrol. 17:932-942.

6. Aydin, Z., van Zonneveld, A.J., de Fijter, J.W., and Rabelink, T.J. 2007. New horizons in prevention and treatment of ischaemic injury to kidney transplants. Nephrol. Dial. Transplant. 22:342-346.

7. Babu, A.N., et al. 2007. Microvascular destruction identifies murine allografts that cannot be rescued from airway fibrosis. J. Clin. Invest. 117:3774-3785.

8. Rabelink, T.J., Wijewickrama, D.C., and de Koning, E.J. 2007. Peritubular endothelium: the Achilles heel of the kidney? Kidney Int. 72:926-930.

9. Woywodt, A., et al. 2003. Elevated numbers of circulating endothelial cells in renal transplant recipients. Transplantation. 76:1-4.

10. Woywodt, A. 2003. Circulating endothelial cells in vasculitis and transplantation. Pathophysiol. Haemost. Thromb. 33:500-502.

11. Kang, D.H., et al. 2002. Role of the microvascular endothelium in progressive renal disease. J. Am. Soc. Nephrol. 13:806-816.

12. Bishop, G.A., Waugh, J.A., Landers, D.V., Krensky, A.M., and Hall, B.M. 1989. Microvascular destruction in renal transplant rejection. Transplantation. 48:408-414.

13. Lagaaij, E.L., et al. 2001. Endothelial cell chimerism after renal transplantation and vascular rejection. Lancet. 357:33-37.

14. Quaini, F., et al. 2002. Chimerism of the transplanted heart. N. Engl. J. Med. 346:5-15.

15. Sidky, Y.A., and Auerbach, R. 1975. Lymphocyteinduced angiogenesis: a quantitative and sensitive assay of the graft-vs.-host reaction. J. Exp. Med. 141:1084-1100.

16. Auerbach, R., and Sidky, Y.A. 1979. Nature of the stimulus leading to lymphocyte-induced angiogenesis. J. Immunol. 123:751-754.

17. Cotran, R.S. 1994. Inflammation and repair. In 
Pathologic basis of disease. R.S. Cotran, V. Kumar, and S.L. Robbins, editors. W.B. Saunders. Philadelphia, Pennsylvania, USA. 51-92.

18. Maiese, K., Li, F., and Chong, Z.Z. 2005. New avenues of exploration for erythropoietin. JAMA. 293:90-95.

19. Chong, Z.Z., and Maiese, K. 2007. Erythropoietin involves the phosphatidylinositol 3-kinase pathway, 14-3-3 protein and FOXO3a nuclear trafficking to preserve endothelial cell integrity. Br. J. Phar- macol. 150:839-850.

20. Fliser, D., Bahlmann, F.H., and Haller, H. 2006. EPO: renoprotection beyond anemia correction. Pediatr. Nephrol. 21:1785-1789.

21. Ferrara, N. 2001. Role of vascular endothelial growth factor in regulation of physiological angiogenesis. Am. J. Physiol. Cell Physiol. 280:C1358-C1366.

22. Reinders, M.E., et al. 2003. Proinflammatory functions of vascular endothelial growth factor in alloimmunity. J. Clin. Invest. 112:1655-1665.
23. Denton, M.D., et al. 2004. TNP-470, an angiogenesis inhibitor, attenuates the development of allograft vasculopathy. Transplantation. 78:1218-1221.

24. Contreras, A.G., Flynn, E., Meyer, E., Sho, M., and Briscoe, D.M. 2007. Anti-angiogenesis therapy interrupts the progression of allograft vasculopathy [abstract]. Am. J. Transplant. 7:331.

25. Lemstrom, K.B., et al. 2002. Vascular endothelial growth factor enhances cardiac allograft arteriosclerosis. Circulation. 105:2524-2530.

\title{
Staying awake puts pressure on brain arousal systems
}

\author{
Giulio Tononi and Chiara Cirelli
}

Department of Psychiatry, University of Wisconsin-Madison, Madison, Wisconsin, USA.

\begin{abstract}
Many brain centers are involved in keeping us awake. One example is the recently discovered hypocretin system located in the posterior hypothalamus. In this issue of the JCI, Rao et al. show that, in mice, synapses targeting hypocretin neurons become stronger when wakefulness is prolonged beyond its physiological duration (see the related article beginning on page 4022). This increase in synaptic strength may be one of the mechanisms that help us to stay awake when we are sleep deprived, but it may also represent one of the signals telling the brain that it is time to sleep.
\end{abstract}

Sleep appears to be a universal phenomenon, since it is present in all species that have been carefully studied so far (1). A lot is known about the brain areas responsible for promoting arousal and the maintenance of wakefulness, as well as those areas crucial for the initiation and maintenance of sleep. The functions of sleep, on the other hand, remain unclear, although it is clear that if wakefulness is prolonged beyond its physiological duration (approximately 16 hours in humans), severe physiological and cognitive deficits occur (2).

\section{What keeps us awake?}

There are several neurochemical systems with diffuse projections that promote and maintain wakefulness. These include the noradrenergic cells in the locus coeruleus (LC); cholinergic cells in the pedunculopontine tegmental nucleus (PPTN), lat-

Nonstandard abbreviations used: LC, locus coeruleus; LDTN, lateral dorsal tegmental nucleus; LTP, long-term potentiation; mEPSC, miniature excitatory postsynaptic current; NREM, non-rapid eye movement; PPTN, pedunculopontine tegmental nucleus; TMN, tuberomamillary nucleus; VTA, ventral tegmental area. Conflict of interest: Giulio Tononi has served as a consultant to Tikvah Therapeutics Inc. Chiara Cirelli has declared that no conflict of interest exists.

Citation for this article: J. Clin. Invest. 117:3648-3650 (2007). doi:10.1172/JCI34250. eral dorsal tegmental nucleus (LDTN), and basal forebrain; histaminergic cells in the tuberomamillary nucleus (TMN) of the posterior hypothalamus; and glutamatergic neurons in various structures in the central nervous system (3). These cells fire at a higher rate during waking than during non-rapid eye movement (NREM) sleep. Dopamine-containing neurons in the substantia nigra and ventral tegmental area (VTA) also modulate arousal. VTA cells fire tonically during quiet wakefulness and NREM sleep, and in bursts (inducing massive release of dopamine) during appetitive waking behavior and REM sleep (4). Psychostimulants, such as amphetamines and cocaine, that block the reuptake of monoamines including norepinephrine, dopamine, and serotonin promote prolonged wakefulness and increase both cortical activation and behavioral arousal (5). The most recent wakefulness-promoting system to be discovered is the hypocretin system. The peptide hypocretin (also known as orexin) is produced by cells in the posterior hypothalamus that provide excitatory input to all remaining wakefulnesspromoting areas, including the LC, PPTN, LDTN, VTA, basal forebrain, and TMN (6) (Figure 1). Hypocretin neurons are most active during waking, especially in relation to motor activity and exploratory behavior, and almost stop firing during both NREM and REM sleep.

Wakefulness-promoting systems appear to be redundant to some extent, which may explain why specific lesions that affect one or a few of these brain areas do not result in a complete and permanent suppression of cortical activation and wakefulness (7). A common mechanism through which these systems produce cortical activation is by closing potassium channels on the cell membrane of cortical and thalamic neurons, thus keeping cells depolarized and ready to fire (8). At sleep onset, wakefulness-promoting neuronal groups are actively inhibited by antagonistic neuronal populations located in the hypothalamus $(9,10)$ and the basal forebrain $(11,12)$, most of which are GABAergic (transmit or secrete $\gamma$-aminobutyric acid). Exactly how the switch from wakefulness to sleep occurs, however, remains unclear.

\section{The homeostatic regulation of sleep}

What is clear is that sleep is regulated through both circadian and homeostatic mechanisms (13). The circadian regulation is responsible for the change in sleep propensity depending on the time of day, with obvious adaptive advantages. Humans, for instance, are a diurnal species (active during the daytime and sleeping mainly at night), while mice and rats are nocturnal and sleep mainly during the day. The homeostatic regulation of sleep, on the other hand, is responsible for the fact that the longer one stays awake, the stronger the pressure to go to sleep becomes (sleepiness increases). Also, the subsequent sleep is 